\title{
Kindesmisshandlung - nicht unfallbedingte Verletzungen bei Kindern
}

\author{
Ralf-Bodo Tröbs, Roberto Gonzalez-Vasquez, Karin Barenberg
}

\section{Zusammenfassung}

Bei der Behandlung verletzter Kinder ist stets die ursächliche Einwirkung einer äußeren körperlichen Gewalt in das differenzialdiagnostische Denken einzubeziehen. Im Verdachtsfall sind die konsequente Aufdeckung der Ursachen einer „non-accidental injury“ und die Ergreifung von Maßnahmen zum zukünftigen Schutz des Kindes erforderlich. Die vorliegende Übersichtsarbeit gibt essenzielle Informationen zu typischen Verletzungsmustern von Haut/Weichteilen, Knochen und Gehirn infolge einer Kindesmisshandlung. Am besten wird man den umfangreichen Erfordernissen der Behandlung betroffener Kinder im Rahmen eines multidisziplinären Teams gerecht.

\section{Child Abuse - Non-Accidental Injuries in Children}

Child abuse has always to be taken into account if babies, toddlers or older children are treated for injuries of the skin/soft tissues, bones and skull or brain. If a non-accidental injury seems probable everything has to be done to determine the cause and to protect the child against further attacks. This review gives essential information to aid in the recognition of typical patterns of skin/soft tissue, bone or brain injury in the case of child abuse. The physician must systematically assess, recognise and act on the indicators for non-accidental injury in collaboration with a multidisciplinary team.

\section{Einleitung}

Die Erkennung der Anwendung von körperlicher Gewalt gehört zu den wichtigen Aufgaben aller Mediziner, die verletzte Kinder behandeln. Dabei erlaubt es die einschlägige Fachkenntnis des Arztes, typische unfallbedingte Verletzungen gegen untypische Verletzungsmuster abzugrenzen und entsprechende diagnostische und sozialmedizinische Maßnahmen einzuleiten. Wünschenswert sind detaillierte Kenntnisse zu Biomechanik, Pathophysiologie und Heilungsverlauf kindlicher Verletzungen. Unerkannte Misshandlungen oder nicht ernst genommene Hinweise darauf können fatale Folgen für das Kind haben, da das Wiederholungsrisiko bei $50 \%$ liegt und einige dieser Leidensgeschichten mit dem Tod enden $[1,3,4]$.

OP-JOURNAL 2010; 26: 78-83

(c) Georg Thieme Verlag KG Stuttgart · New York DOI http://dx.doi.org/10.1055/s-0030-1250172
Dies zeigt, dass der Verdacht auf eine Kindesmisshandlung dem Behandlungsteam ein hohes Maß an Verantwortung aufbürdet. So ist es weiterhin wissenschaftlich gesichert, dass misshandelte Kinder ein Leben lang unter der Last zu tragen haben. Zu den Spätfolgen zählen mentale, psychische und körperliche Leiden. So werden beim Erwachsenen verstärkt Herz- und Lebererkrankungen, Depressionen, Angstzustände, Suchtkrankheiten sowie Arbeitslosigkeit und unerwünschte Schwangerschaft registriert [5].

\section{Lebenslange Folgen nach Kindesmisshandlung}

In einer bahnbrechenden Publikation prägten Kempe und Koautoren (1962) den Begriff des „battered child“-Syndroms (engl. battered = zerschlagen, misshandelt), der sich auch im deutschen Sprachraum einbürgerte [5]. Entsprechend der Breite des Spektrums an Schädigungen (Abb. 1), die nicht nur ein- zelne körperliche Schäden umfasst, sondern komplexe Auswirkungen auf die gesamte Entwicklung des Kindes haben kann, verwendet man heute den Terminus „nicht akzidentelle Verletzung“ (engl. non accidental injury = nicht zufällige Verletzung). Trotz einschlägiger medizinischer Weiterbildungsmaßnahmen ist bei der Aufdeckung von Kindesmisshandlungen auch heute noch von einer beträchtlichen Dunkelziffer auszugehen.

Die nachfolgende Übersicht beschäftigt sich insbesondere mit der körperlichen Misshandlung von Kindern und Jugendlichen, die sich meist im familiären Umfeld des Kindes abspielt. Davon abzugrenzen sind der sexuelle Missbrauch, die seelische Vernachlässigung sowie das Münchhausen-Stellvertreter-Syndrom, welche nur mittelbar Gegenstand nachfolgender Ausführungen sind.

\section{Hintergrund}

Obwohl Kindesmisshandlungen in allen sozioökonomischen Schichten vorkommen, gibt es spezielle Prädispositionen bzw. Risikofaktoren. Dazu gehören Eltern, die als Kind selbst missbraucht wurden, Drogen- und Alkoholkonsum in der Familie sowie bspw. Krisensituationen und finanzieller Druck auf die Familie. Besonders gefährdet sind Kinder mit einer vorbestehenden Behinderung, sog. „Schreikinder“, sowie Geschwister bereits misshandelter Kinder. Die meisten körperlichen Misshandlungen betreffen Kinder im Alter unter 3 Jahren, während ein sexueller Missbrauch überwiegend die Altersgruppe über 6 Jahre betrifft.

Insbesondere bei Verletzungen im Vorlauflernalter Unfallhergang hinterfragen.

\section{Anamnese}

Die sorgfältige Anamnese ist ein entscheidender Schlüssel für die Aufdeckung nicht akzidenteller Verletzungen. Eine Kernfrage liegt darin, ob der 


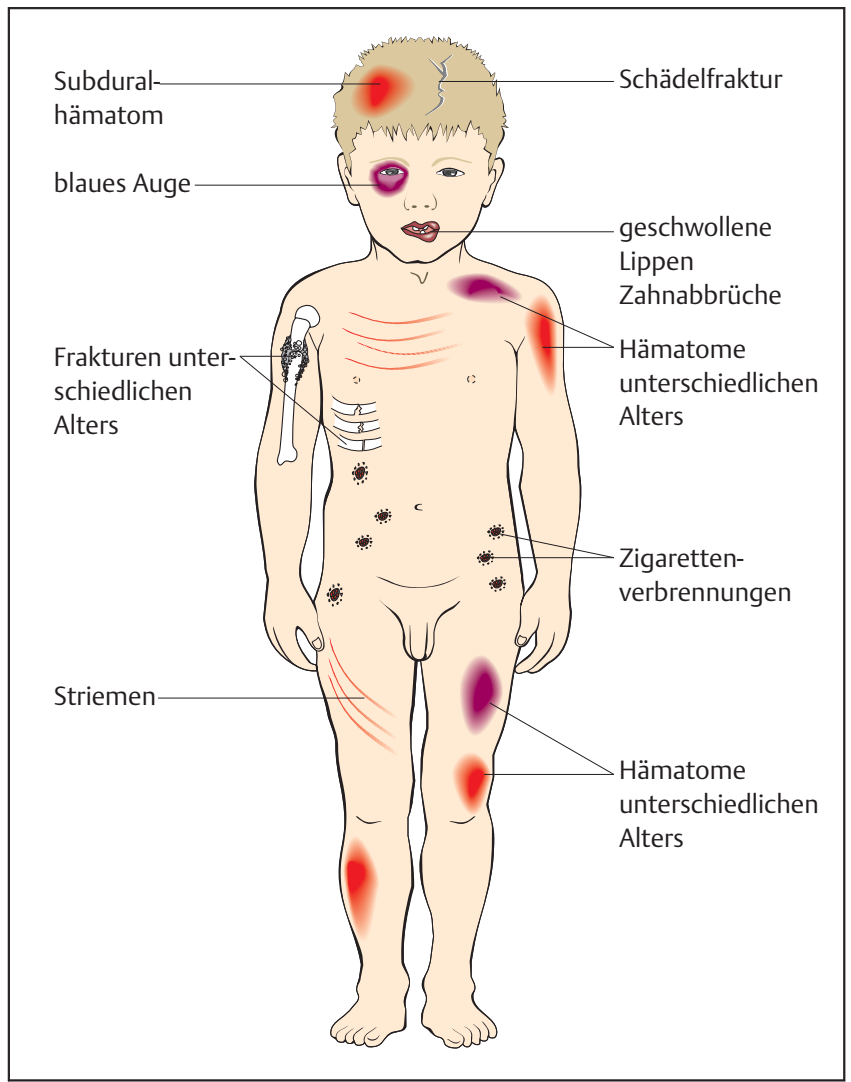

angegebene Unfallmechanismus adäquat für die vorgefundene Verletzung des Kindes ist (Plausibilitätsfrage). Weiterhin sind die Schwangerschafts- und $\mathrm{Ge}$ burtsanamnese sowie die Fahndung nach familiären Erkrankungen von besonderer Bedeutung.

Zur Eingrenzung des Ereignisses kann die Elternbefragung in $\mathbf{4}$ „W“ gegliedert werden:

- Wie entstand die Verletzung?

- Wann trat das Ereignis ein?

- Wer verursachte die Verletzung?

- Warum kam es zum Ereignis?

Nach Herrmann [3] ergeben nachfolgende Sachverhalte einen Anhalt für das Vorliegen einer nicht akzidentellen Verletzung:

- fehlende, vage, unklare oder wechselnde Erklärungen zu Unfallursache und -hergang

- für Alter bzw. Entwicklungsstand inadäquater Unfallhergang

- verzögertes Aufsuchen des Arztes

- schwere Verletzungen, angeblich durch das Kind selbst oder Geschwister zugefügt

- Entdeckung zusätzlicher, zuvor nicht angegebener Verletzungen
Abb. 1 Synopsis unterschiedlicher Verletzungen nach Misshandlung.

- wiederholte Verletzungen mit unklarem Hergang und gehäuftem Wechsel des Arztes

- Hinweise von Dritten oder vom Kind selbst

Hämatome können im Zusammenhang mit einer vorbestehenden angeborenen (Bluterkrankheit) oder erworbenen Gerinnungsstörung auftreten. So kann bspw. eine fehlende Vitamin-K-Prophylaxe beim Neugeborenen eine spontane Blutung hervorrufen. Unter Umständen ist eine Spezialdiagnostik erforderlich. Weiterhin kann bspw. eine Hirnblutung infolge eines schweren Faktor-VIII-Mangels eintreten, was nur durch eine Messung der Faktor-VIII-Aktivität beweisbar ist. Ähnliche Spezialuntersuchungen ergeben sich bei der von-Willebrand-Erkrankung oder bei Thrombozytenfunktionsstörungen [8].

Bei Frakturen im Zusammenhang mit banalen Anlässen ist an das Vorliegen der Glasknochenkrankheit (Osteogenesis imperfecta) zu denken. Diese kann bei negativer Familienanamnese auch infolge einer Spontanmutation auftreten. Bei diesen Kindern kommt es ohne adäquates Trauma zu Knochenbrüchen, die oft nur von einer minimalen Weichteilschwellung begleitet sind. Typische kli- nische Zeichen der Osteogenesis imperfecta sind eine besondere Schädelkonfiguration, blaue Skleren, eine Überbeweglichkeit der Gelenke, brüchige Zähne sowie grazile Knochen im Röntgenbild. Weitere Differenzialdiagnosen betreffen Knochenbrüchigkeit nach Frühgeburt, Rachitis, Hypervitaminose A, Osteodystrophie infolge chronischer Nieren-, Leber- oder Darmerkrankungen, Kupfermangel und andere.

\section{Diagnostikfahrplan}

Die folgerichtige Diagnostik sowie Einordnung und Dokumentation der Befunde sind entscheidend für den Erfolg ärztlichen Handelns. Neben der sorgfältigen klinischen Ganzkörperuntersuchung gehören eine adäquate Bildgebung, ausgewählte Laboruntersuchungen sowie die Gewinnung und Aufbewahrung forensisch wichtigen Materials zum Aufgabengebiet des Arztes. Die Untersuchung des Augenhintergrunds zum Ausschluss einer Netzhautblutung bzw. einer Stauungspapille ist obligat. Ein rechtsmedizinisches Konsil gibt zusätzliche Sicherheit und Handlungsfreiheit. Tab. 1 gibt einen Überblick über die diagnostischen Schritte bei Misshandlungsverdacht.

Das Babygramm erlaubt keine ausreichende Skelettdiagnostik!

\section{Röntgen, Ultraschall- und Schnittbilduntersuchungen}

Die Erfassung möglichst aller knöchernen Verletzungen und Verletzungsfolgen erfordert ein Röntgenscreening des Skeletts. Geröntgt werden Schädel und Wirbelsäule im anterior-posterioren sowie im seitlichen Strahlengang. Brustkorb, knöchernes Becken sowie die Extremitäten werden in einer Ebene geröntgt. Bei Verletzungsverdacht wird gezielt in einer 2. Ebene nachgeröntgt. Die frühe übliche Ganzkörperröntgenaufnahme des Säuglings, das Babygramm, sollte nicht mehr angefertigt werden, da es unter Umständen weniger gravierende Frakturen oder periostale Reaktionen verschleiert.

Einrisse und Prellungen von Leber, Milz, Nieren und u.U. Pankreas mit begleitender Ansammlung von Blut oder Flüssigkeit in der Bauchhöhle können mittels Ultraschall erfasst werden. Schnittbilduntersuchungen vervollkommnen im Bedarfsfalle die bildgebende Diagnostik. 
Tab. 1 Diagnostik.

\begin{tabular}{|c|c|c|}
\hline Maßnahme & Inhalt & Bemerkungen \\
\hline Anamnese & $\begin{array}{l}\text { genauer Unfallhergang } \\
\text { soziale und familiäre Verhältnisse }\end{array}$ & \\
\hline $\begin{array}{l}\text { körperliche } \\
\text { Untersuchung }\end{array}$ & $\begin{array}{l}\text { Ganzkörperuntersuchung } \\
\text { Allgemein- und Ernährungszustand } \\
\text { Körpergewicht und -länge }\end{array}$ & $\begin{array}{l}\text { entkleidetes Kind } \\
\text { Inspektion der Mundhöhle sowie } \\
\text { genital-perianal }\end{array}$ \\
\hline Bildgebung & $\begin{array}{l}\text { Skelett } \\
\text { - Schädel und Wirbelsäule in } \\
2 \text { Ebenen } \\
\text { - lange Röhrenknochen, Thorax } \\
\text { und Becken in } 1 \text { Ebene } \\
\text { Abdomen } \\
\text { - Sonografie } \\
\text { Zentralnervensystem } \\
\text { - Schädel-CT }\end{array}$ & $\begin{array}{l}\text { Kein Babygramm! } \\
\text { Ggf. 2. Ebene zur Frakturbestäti- } \\
\text { gung. } \\
\text { Im Zweifelsfalle Szintigrafie zur } \\
\text { Darstellung von Rippenfrakturen. } \\
\text { Nachfolgend MRT Schädel zur Be- } \\
\text { urteilung von Spätfolgen. }\end{array}$ \\
\hline Labor & $\begin{array}{l}\text { Blutbild } \\
\text { Gerinnung (Quick, PPT, Blutungs- } \\
\text { zeit, spezial) } \\
\text { Leber (GOT, GPT, Y-GT) } \\
\text { Niere (Kreatinin) } \\
\text { Pankreas (Lipase) } \\
\text { Knochen (Kalzium, Phosphat, AP) } \\
\text { Urinstreifentest (Erythrozyten i.U.) }\end{array}$ & $\begin{array}{l}\text { Ausschluss Glutarazidurie bei intra- } \\
\text { kraniellen Befunden } \\
\text { u.U. spezielle Gerinnungsdiagnos- } \\
\text { tik }\end{array}$ \\
\hline $\begin{array}{l}\text { fachüber- } \\
\text { greifend }\end{array}$ & $\begin{array}{l}\text { Beurteilung des Augenhintergrunds } \\
\text { kinderradiologisches und ggf. } \\
\text { rechtsmedizinisches Konsil }\end{array}$ & \\
\hline $\begin{array}{l}\text { Bilddokumen- } \\
\text { tation }\end{array}$ & $\begin{array}{l}\text { Fotos mit Maßstab, Skizzen, } \\
\text { Farbbeschreibung }\end{array}$ & \\
\hline
\end{tabular}

\section{Labordiagnostik}

Die begleitende Labordiagnostik dient zum einen der Suche nach Verletzungen innerer Organe (Leber, Bauchspeicheldrüse). Andererseits ist es geboten, $\mathrm{Er}$ krankungen auszuschließen, die eine äußere Gewaltanwendung auf das Kind vortäuschen könnten. Hierzu dienen die laborchemische (Gerinnungsstatus) und klinische Überprüfung (Blutungszeit) der Gerinnung sowie bei Frakturen eine orientierende Abklärung des Kalziumund Phosphatstoffwechsels.

„Normale“ blaue Flecken finden sich im Lauflernalter an der Stirn, über den vorderen Darmbeinstacheln und an den Vorderseiten der Unterschenkel.

\section{Haut und Weichteile}

Hämatome von Haut und Unterhaut gehören zu den am besten sichtbaren äußeren Stigmata einer stattgehabten Gewalteinwirkung (Abb.2). Sie sind bei $9 / 10$ der betroffenen Kinder zu finden. Hämatome junger und weitgehend immobiler Säuglinge in jeglicher Lokalisation bedürfen der Erklärung. Mit dem Erlernen des Laufens stürzen und stoßen sich Kinder gehäuft, was naturgemäß zu Hautunterblutungen an charakteristischen Stellen führt. Prädilektionsstellen für diese akzidentellen Hämatome sind Stirn und Schläfen, die vorderen Beckenkammstachel (Spina iliaca anterior superior) sowie beide Schienbeine. Andererseits sollten unfalluntypische Hämatome einer kritischen Überprüfung unterzogen werden. Als nicht akzidentelle Hämatomlokalisationen gelten Hämatome des Gesichts (Orbita, Wangen, Ohren, Kiefernwinkel), des Körperstamms (Schulter, Brustkorb, Bauch, Rücken inklusive der rückwärtigen Oberschenkel), der Unterarme (Parierbewegung) sowie des Genitales. Die Form der Unterblutungen kann weiterhin Anhaltspunkte über die Art der einwirkenden Gewalt geben (Handabdruck, Würgemal, Seil- oder Stockabdruck, Bissmarke).

\section{Verletzungen des Gesichts, der Mundhöhle und der Zähne}

Ca. die Hälfte der misshandelten Kinder weist nicht akzidentelle Verletzungen von Gesicht und Kopf auf. Über den Mund nimmt das Kind Nahrung zu sich oder es gibt Lautäußerungen von sich. Hier können Spannungen zu den Eltern

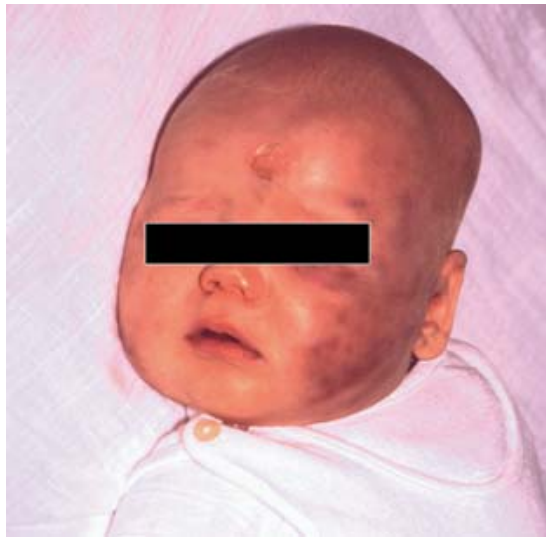

Abb. 2 Multiple flächige Hämatome und Brandverletzung der Stirn infolge Gewalt.

ihren Ursprung nehmen. So treten Verletzungen von Lippen, Lippenbändchen, Zähnen und Mundhöhle, z.B. infolge frustraner Fütterungsversuche, infolge von Schlägen oder Einrammen der Flasche auf. Auch Zahnabbrüche, -frakturen oder Impressionen gehören in diese Gruppe [1,3,7].

Bestimmte thermische Verletzungen beim Säugling und Kleinkind sind misshandlungsverdächtig.

\section{Eintauchverbrühungen und Kontaktverbrennungen}

Auch thermische Verletzungen der Haut können infolge einer Misshandlung auftreten. Unterschieden werden Verbrühungen durch heiße Flüssigkeiten oder Verbrennungen infolge des Kontakts mit Flammen oder Festkörpern. Typisch sind Eintauchverletzungen (Immersionsverletzungen), die davon resultieren, dass Hand oder Fuß des Kindes gewaltsam in die heiße Flüssigkeit getaucht werden. Es resultieren Verbrühungswunden, die die gesamte Zirkumferenz des eingetauchten Gliedmaßenabschnitts handschuh- oder strumpfartig umgreifen (engl. stocking glove burn). Verbrühungen der Geschlechtsorgane und deren Umgebung sowie des Gesäßes sind ebenfalls bis zum Nachweis des Gegenteils als nicht akzidentell zu werten. Wenn Kinder gewalttätig in eine heiße Flüssigkeit getaucht werden, führt das reflexartige Anziehen der Gliedmaße zu charakteristischen Aussparungen in der Beugefalte (z.B. am Knie).

Typische akzidentelle Verbrennungen des Kleinkinds, das die Umwelt sehend und tastend erschließt, betreffen die Fingerkuppen sowie die Palmarflächen von Fingern und Hand. 


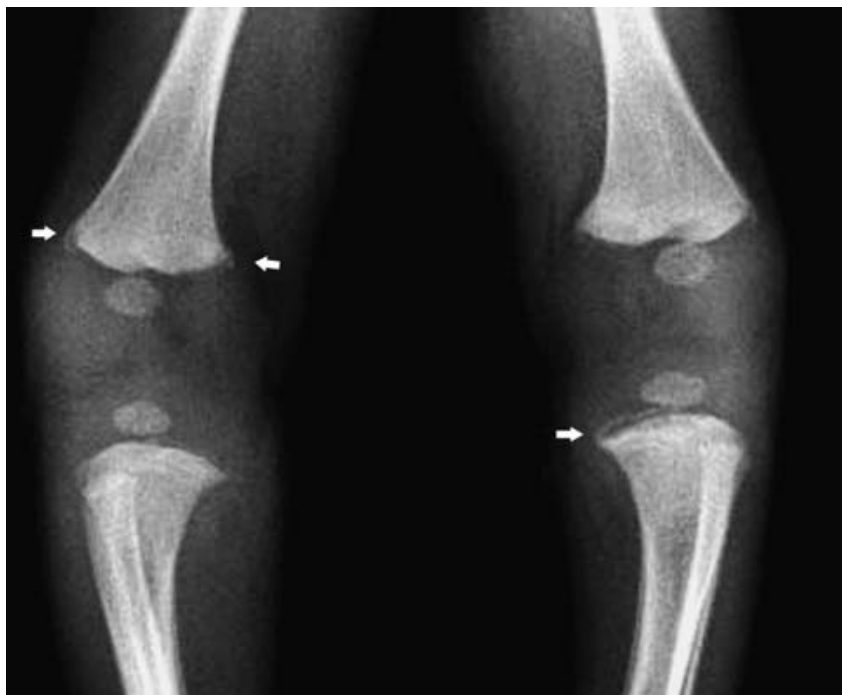

Tab. 2 Spezifität unterschiedlicher Frakturen für das Vorliegen einer Kindesmisshandlung.

\begin{tabular}{ll}
$\begin{array}{ll}\text { Spezi- } \\
\text { fität }\end{array}$ & $\begin{array}{l}\text { Frakturtyp, -muster, } \\
\text {-lokalisation }\end{array}$ \\
\hline hoch & - Brüche im Vorlauflernalter \\
& (auch Oberschenkel und Ober- \\
& armschaft) \\
& - metaphysäre Brüche \\
& - Knochenbrüche unterschied- \\
& lichen Alters \\
- & Rippenbrüche \\
- & Schulterblattbrüche \\
& - laterale/mediale Schlüsselbein- \\
& brüche \\
& - Wirbelbrüche \\
& - Fingerknochenbrüche bei nicht \\
& mobilen Kindern \\
- & beidseitige Knochenbrüche \\
- & komplexe Schädelbrüche \\
\hline gering & Schlüsselbeinbrüche in loco \\
& typico \\
- & einseitige Brüche langer Kno- \\
& chen im Laufalter \\
- & lineare Schädeldachfrakturen
\end{tabular}

Sogenannte Kontaktverbrennungen spiegeln das geometrische Muster der Oberfläche der Verbrennungsquelle wider. Absichtlich zugefügte Zigarettenverbrennungen können anhand tiefer und irregulär-rundlicher Abdrücke von etwa $8 \mathrm{~mm}$ Durchmesser, insbesondere an Hand- oder Fußrücken sowie in der Unterleibsregion erkannt werden. Diese Wunden hinterlassen Narben. Auch sind geformte Verbrennungsabdrücke von Herdplatten, Heizkörpern, Bügeleisen usw. ebenfalls hochgradig verdächtig.
Abb. 3 Misshandlungstypische beidseitige metaphysäre Femur- sowie Tibiafrakturen eines Säuglings.

\section{Frakturen}

Knochenbrüche beim Kind sind stets das Resultat einer erheblichen äußeren Gewalteinwirkung.

Die Heilung eines kindlichen Knochenbruchs folgt einem bestimmten zeitlichen Muster (modif. nach [4]):

- Rückbildung der Weichteilschwellung nach 4-10 Tagen (Schwankung 2-10 Tage)

- zarte periostale Kallusbildung nach 10-14 Tagen (Schwankung 4-21 Tage)

- Verwischen der Frakturlinien im Röntgen nach 14-21 Tagen (Schwankung 10-21 Tage)

- fester Kallus besteht nach 21-42 Tagen (Schwankung 14-90 Tage)

- Remodelling-Prozesse benötigen 3 Monate bis 2 Jahre

Bestimmte Frakturmuster und -typen sind besonders verdächtig auf das Vorliegen eines nicht akzidentellen Entstehungsmechanismus (Tab. 2). Knapp die Hälfte aller misshandelten Kinder weist 1 oder mehrere Knochenbrüche auf. So zeigen die Abb. $\mathbf{3}$ und $\mathbf{4}$ Frakturen eines 2 Monate alten dystrophen Säuglings aus schwierigem sozialen Umfeld nach nicht akzidentellen Verletzungen. Charakteristisch ist das gleichzeitige Auftreten von Brüchen unterschiedlicher Lokalisationen und Heilungsstadien. Im Zusammenhang mit einer körperlichen Misshandlung werden Knochenbrüche, insbesondere in den ersten 3 Lebensjahren angetroffen. Besonders im Säuglingsalter - diese Kinder können noch nicht laufen und werden erst passiv durch die Eltern mobil - ist in einem hohen Prozentsatz ein nicht akzidentelles Trauma Frakturursache. Echt unfallbedingte, d.h.

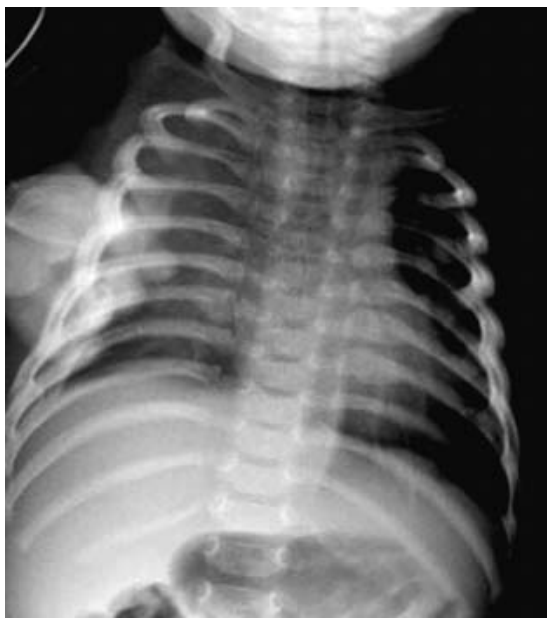

Abb. 4 Dorsale verheilte Rippenserienfrakturen beiderseits sowie Klavikulafraktur links.

akzidentelle Knochenbrüche sind dagegen eine Domäne des älteren Kindes.

Beim Säugling und Kleinkind sind Frakturen im Bereich der Wachstumsfugen (epi- und metaphysäre Frakturen) langer Röhrenknochen Folge einer erheblichen Gewalteinwirkung. Bildlich werden die Frakturtypen als Eck- bzw. Korbhenkelfrakturen beschrieben. Eckfrakturen sind die Folge von Zug und Verbiegung der Extremität mit Anspannung des Periosts und Perichondriums über der Extremität. Daneben treten lineare Stauchungsfrakturen misshandlungsbedingt in Erscheinung.

In den Vereinigten Staaten von Amerika werden $1 / 3$ aller Oberschenkelfrakturen von Kindern im Alter unter 5 Jahren und 2/3 Frakturen bei Kindern unter 1 Jahr einem nicht akzidentellen Trauma zugeordnet (Oppenheim und Bowen, 2007 [9]).

Rippenfrakturen im seitlichen und hinteren Abschnitt sind ebenfalls hoch verdächtig. $\mathrm{Zu}$ ihrer Erkennung benötigt man eine Röntgenaufnahme des knöchernen Thorax. Ein Standardröntgenthorax, wie er in der Lungendiagnostik Verwendung findet, ist nicht ausreichend und irreführend. Im Zweifelsfalle dient eine Knochenszintigrafie der Diagnosebestätigung.

Neben Frakturen im engeren Sinne können diaphysäre periostale Reaktionen („verkalkte subperiostale Hämatome“) insbesondere beim Säugling die Einwirkung einer lokalen Gewalt anzeigen. Das anatomische Substrat für diese Veränderungen ist die nur lockere Anhaftung der Knochenhaut im Bereich des Knochen- 
schafts (Diaphyse), sodass diese infolge lokaler Verdrehung und Abscherung vom Knochen abgelöst werden kann. Dies hat eine subperiostale Einblutung zur Folge, die unter Verkalkung ausheilt. Im metaphysären Bereich dagegen ist die Knochenhaut innig mit der Knochenoberfläche verwoben.

\section{Frakturen des Schädeldachs}

Banale Stürze, bspw. von Bett, Couch oder Wickeltisch, führen in knapp $5 \%$ der Fälle zu einfachen Schädeldachfissuren, die häufig parietal lokalisiert sind. Klaffende Frakturen mit einer Länge über $5 \mathrm{~cm}$, Mehrfachfrakturen sowie verzweigte Frakturlinien können dagegen nicht so einfach auf einen Sturz aus minderer Höhe zurückgeführt werden. Abb. 5 zeigt die parietale Schädeldachfraktur links eines 9 Monate alten Säuglings, bei dem lediglich eine Schwellung beobachtet worden sei, jedoch kein Unfall eruiert werden konnte. Das Gleiche betrifft Impressionsfrakturen des Hinterhaupts und insbesondere schwerere unfallbedingte Hirnveränderungen. In diesen Fällen ist der Verdacht auf eine schwere nicht akzidentelle Gewalteinwirkung gegeben. Deshalb muss das Unfallgeschehen in diesen Fällen kritisch hinterfragt werden.

Neurologische Symptome, paravertebrale Rippenbrüche und Einblutungen in die Netzhaut des Auges sind beweisend für ein „Schütteltrauma“.

\section{Schüitteltraumasyndrom}

Das Schütteltrauma (syn. shaken baby syndrome, whiplash shaken infant u.a.) bewirkt irreversible und schicksalsbestimmende Beeinträchtigungen des schutzlosen Säuglings oder Kleinkinds $[1,3]$. Es handelt sich um eine besonders perfide Art der Misshandlung.

Das Geschehen besteht darin, dass der Säugling an den Oberarmen oder am Brustkorb gepackt wird und gewaltsam auf das Heftigste hin- und her geschüttelt wird. Man spricht auch von einem Akzelerations-Dezelerations-Trauma. Dabei bewirken Rotations- und Scherkräfte zwischen Schädelknochen und Hirnoberfläche sowie innerhalb des Gehirns nicht nur das Zerreißen der zwischen weißer Hirnhaut und Hirnoberfläche gelegenen Brückenvenen. Vielmehr kommt es zum Abscheren von Nervenverbindungen am Übergang zwischen grauer und weißer Hirnsubstanz. Man spricht

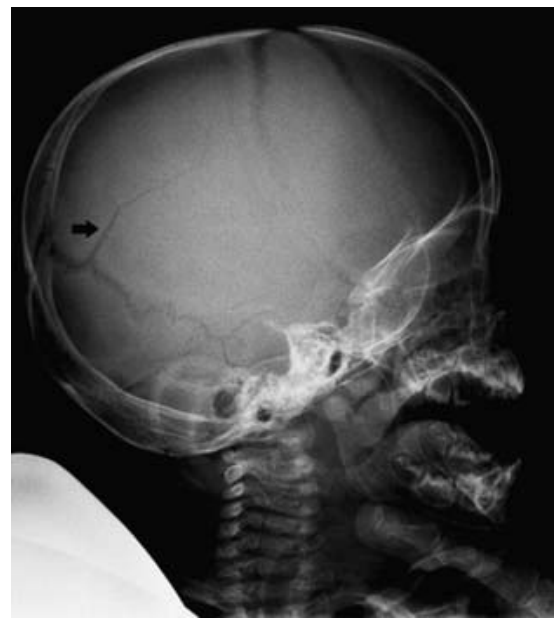

Abb. 5 Parietale Schädeldachfraktur links eines 9 Monate alten Säuglings.

von einem diffusen axonalen Trauma. Diese Schäden, verbunden mit Störungen der Hirndurchblutung und -sauerstoffversorgung, resultieren weiterhin in einer diffusen Hirnschwellung mit begleitender Erhöhung des Schädelbinnendrucks. Die Entstehung von Blutungen der Netzhaut des Auges sowie Glaskörperblutungen kann ebenfalls auf die Einwirkung von Scher- und Rotationskräften innerhalb des Augapfels sowie auf eine Erhöhung des intrakraniellen Hirndrucks zurückgeführt werden. Die Schwere der Verletzung führt zu sofortiger Beeinträchtigung des Kindes - ein symptomloses Intervall existiert nicht. Es ergibt sich das Nebeneinander subduraler Einblutungen ins Gehirn, von Netzhautblutungen sowie unter Umständen von Oberarmknochen-, Rippen- sowie anderer Frakturen der Röhrenknochenmetaphyse. Betroffen sind insbesondere Säuglinge im sog. physiologischen „Hauptschreialter“, jedoch auch ältere Kinder.

Klinisch zeigen die Säuglinge schwere neurologische Symptome wie Bewusstseinseintrübung, Krämpfe, erhöhte Reizbarkeit, Atemstillstände und Temperaturregulationsstörungen. Unspezifische Symptome, wie Trinkschwäche oder Erbrechen, können zu einer Fehleinschätzung der zugrunde liegenden Ursache und damit zur Verzögerung der notwendigen Intervention führen.

Die Diagnose wird durch ein initiales Computertomogramm von Schädel und Gehirn gesichert. Zur Verlaufskontrolle und Einschätzung des Spätresultats erfolgt die Kernspinuntersuchung nach 5 Tagen sowie nach 2-3 Monaten. Die

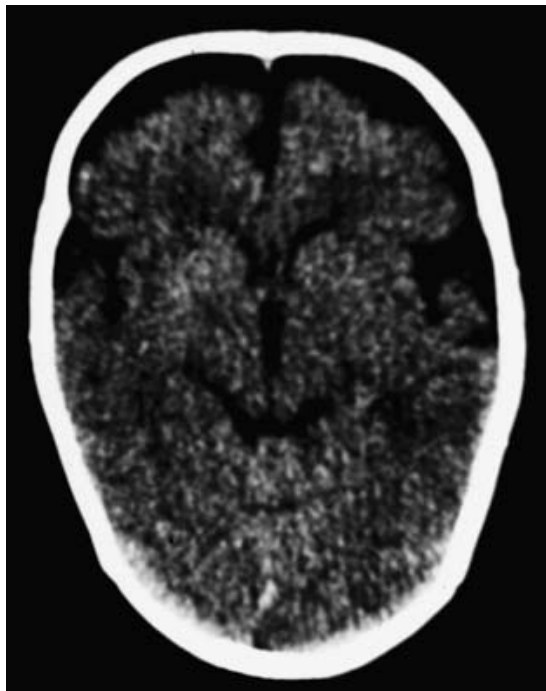

Abb. 6 Frontale Hirnatrophie nach Misshandlung (Computertomogramm).

mittels Bildgebung sichtbaren Folgen können in zystischen Hirnveränderungen (multizystische Enzephalopathie, Porenzephalie), Hirnschrumpfung mit Ausbildung subduraler Hygrome (Abb. 6) sowie einem ausbleibenden Kopfwachstum (Mikrozephalie) bestehen.

Das Schütteltrauma ist von einer hohen Letalitätsrate begleitet. Überlebende Kinder leiden nach einem Schütteltrauma zu einem überwiegenden Anteil lebenslang an den gravierenden Folgen, die psychomotorische Störungen, Krampfleiden, spastische Lähmungen, Seh-, Hörund Sprachausfälle betreffen können.

\section{Was tun?}

Alle Bemühungen müssen darauf gerichtet werden, eine Wiederholung der Misshandlung zu vermeiden. Dabei rangiert die Hilfe für die betroffene Familie vor der Strafe.

Entsprechend der im Bürgerlichen Gesetzbuch verankerten Richtlinien zur Ausübung der elterlichen Sorge haben Kinder in Deutschland ein Recht auf gewaltfreie Erziehung. Dieses Recht bezieht sich sowohl auf körperliche Bestrafungen als auch auf seelische Grausamkeiten oder einen entwürdigenden Umgang. Mit Übernahme der Behandlung eines betroffenen Kindes ist der Arzt der Sachwalter für dessen Interessen. Er hat im Bedarfsfalle aktiv einer zukünftigen Kindeswohlgefährdung entgegenzutreten. In vielen Fällen wird die Wahrnehmung der kindlichen Interessen formal im Widerspruch zur ärztlichen Schwei- 
gepflicht stehen. Hier ist das Kindeswohl das höhere Gut.

Um den vielfältigen Erfordernissen im Gefolge einer Misshandlung gerecht zu werden, ist die Gründung einer Kinderschutzgruppe unter Einbeziehung von Ärztinnen, Pflegerinnen, Psychologen und sozialen Mitarbeitern der beste Weg. Der pflegenden Bezugsschwester kommt im Prozess der Problemerfassung eine wesentliche Rolle $\mathrm{zu}$ [2].

Eine Kindesmisshandlung ist ein Offizialdelikt, sodass eine Information der Staatsanwaltschaft erfolgen soll. Diese veranlasst weitere Ermittlungen und leitet unter Umständen ein Strafverfahren ein [1].

Unabhängig davon ist es sinnvoll, das Jugendamt zur Abschätzung des Gefährdungsrisikos eines Kindes einzuschalten. Dieses ist gesetzlich verpflichtet, entsprechende Hilfestellungen zu leisten. Die Einschaltung eines Rechtsmediziners kann bestehende Vermutungen erhärten und fachlich untermauern.

\section{Literatur}

${ }^{1}$ AWMF online. Kindesmisshandlung und Vernachlässigung. S2-Leitlinie. Leitlinie der Deutschen Gesellschaft für Sozialpädiatrie und Jugendmedizin, übernommen von der Dt. Ges. f. Kinderheilkunde und Jugendmedizin und der Dt. Ges. f. Kinderchirurgie; 9/2008

${ }^{2}$ Fahr S. Kindesmisshandlung im Berufsalltag von Pflegeeltern. Kinderkrankenschwester 2009; 28: 332-336

${ }^{3}$ Herrmann B. Körperliche Misshandlung von Kindern. Somatische Befunde und klinische Diagnostik. Monatsschr Kinderheilkd 2002; 150: 1324-1338

4 Jayakumar P, Barry M, Ramachandran M. Orthopaedic aspects of paediatric non-accidental injury. J Bone Joint Surg [Br] 2010; 92: 189-195

5 Jenny C. Medicine discovers child abuse. JAMA 2008; 300: 2796-2797

${ }^{6}$ Kempe CH, Silverman FN, Steele BF et al. The battered-child syndrome. JAMA 1962; 181: $17-24$

7 Nuzzolese E, Lepore MM, Montagna F. Child abuse and dental neglect: the dental team's role in identification and prevention. Int J Dent Hygiene 2009; 7: 96-101
8 Olivieri M, Kurnik K, Bidlingmaier C. Coagulation testing in the evaluation of suspected child abuse. Hämostaseologie 2009; 29: 190-192

9 Oppenheim WL, Bowen RE. Child abuse. In: Staheli LT, Song KM, eds. Pediatric orthopaedic secrets. 3rd ed. Philadelphia: Mosby/ Elsevier; 2007: 123-129

Prof. Dr. med. Ralf-Bodo Tröbs Direktor

Dr. med. Roberto Gonzalez-Vasquez Oberarzt

\section{Karin Barenberg}

Oberärztin

Kinderchirurgische Klinik Marienhospital Herne, Stiftung Katholischen Rechts Ruhr-Universität Bochum Widumer Straße 8 44627 Herne ralf-bodo.troebs@ marienhospital-herne.de 